\title{
RETRIEVAL OF IMAGES USING SVM
}

\author{
J.V. Shiral ${ }^{1 *}$, Munmun Burman ${ }^{2}$, Apurva Bhadbhade ${ }^{3}$, Dhanashree Patil ${ }^{4}$, Kajal Motghare $^{5}$, Neha Wanjari $^{6}$ \\ $1 *$ Asst. Professor DBACER,Nagpur \\ 2,3,4,5,6 Dept. of CSE, DBACER,Nagpur
}

*Corresponding Author: -

\begin{abstract}
: -
Image retrieval is a technique which is used to search and retrieve images from a large database of digital images. Content-based image retrieval (CBIR) is a technique which allows searching images from large scale image database based on contents as needed by user.This paper introduces a technique to retrieve images by classifying it on the basis of the features and characteristics it contains using Support Vector Machine (SVM). The dataset of images is created which is used for feature matching purpose by SVM to find similar images from the database and based on user requirements images are retrieved.
\end{abstract}

Keywords: -SVM, dataset, image classification, image retrieval, CBIR, feature matching.

\section{(c) $(\$)$ (i)}




\section{INTRODUCTION}

Digital image is a representation of a two-dimensional image as a finite set of digital values, called picture elements or pixels. Image retrieval is a process for retrieving images from a large database of digital images. The process of retrieving desired images from a large collection on the basis of features that can be automatically extracted from the images using SVM. These systems are known as CBIR (Content based image retrieval) have received intensive attention in the literature of image retrieval since this area was started years ago, and thus remains many challenging research problems that continue to attract researchers from multiple disciplines. Before introducing the fundamental concept of image retrieval, let us take a brief look at SVM. Support Vector Machine (SVM) is a supervised learning model associated with some learning algorithms that analyze data and recognize patterns used for classification and retrieval.Support Vector Machines were developed to solve classification problems. A support vector machine (SVM) is a method of machine learning that attempts to take input data and classify it into one of the categories. SVM requires a set of training examples that belongs to one or more categories.SVM is an input/output machine. A user is able to put an input image and depending upon the model developed through training SVM returns an output.SVM equips a greater ability to generalize which is the goal in image retrieval process.

\section{LITERATURE REVIEW}

A method called classification-driven biomedical image retrieval framework based on image filtering and similarity fusion is done by using supervised learning techniques. In this method, the probabilistic outputs of a multiclass support vector machine (SVM) classifier as category prediction of query and database images are exploited at first to filter out unrelated images, hence reducing the search space for matching similar images. Images are classified at a global level according to their modalities based on different lowlevel, concept. It is difficult to find a common feature to compare images efficiently for the given queries [2].

Another method shows that support vector machines (SVM's) can generalize well on difficult image classification problems where the only features are high dimensional histograms.Heavy-tailed RBF kernels are evaluated on the classification of images extracted from the Corel stock photo collection and shown to far outperform traditional polynomial or Gaussian radial basis function (RBF) kernels. From classification trees to neural networks, there are manypossible choices for what classifier to use. The support vector machine (SVM) approach is considered a good candidatebecause of its high generalization performance without the need to add advance knowledge, even if the dimension of the input image is very high [4].

Recently there has been a lot of research in classifiers that have good generalizationperformance by maximizing the margin. Examples of such classifiers are boosting andSupport Vector Machines (SVM) [1]. Using boosting provides a good theoretical and practical convergence to a low error rate in little iteration, its speediness being one of the major advantages over other algorithms such as SVM. Furthermore, boosting can also be used as a feature selection algorithm.

\section{METHODOLOGY}

Content-based image retrieval (CBIR) is a technique which uses visual contents to search images from large scale image databases according to user's interests.CBIR techniques can be grouped into two main types as supervised and unsupervised.

Supervised technique relies on having example pattern or feature vectors which have already been assigned to a defined class.

Unsupervised technique does not rely on possession of existing examples from a known pattern class.

In this method system uses SVM which is a supervised model.This method will be able to retrieve images by matching the features of an input image and images of database using SVM.

In this method following modules are used:

- Dimensionality Reduction

- Feature Extraction

- SVM processing

- Image retrieval

\section{1) Dimensionality Reduction}

Dimensionality reduction is the transformation of high dimensional data into meaningful representation of reduced dimensionality of standard size. This system needs a standard size to proceed which is $384 * 256$ in general.

\section{2) Feature extraction}

This paper defines feature extraction as a function of various measurements, each of which specifies some experimental property of an object, and is computed in such a way that it quantifies some significant characteristics of the object. Various factors on which feature extraction depends are as follows:

\section{a. Color}

The color feature is one of the most famous visual feature in image retrieval process. Humans perceive color as a combination of three colors as: Red, Green, and Blue which creates a color space. $R G B$ colors are called as primary colors and are additive. 


\section{b. Shape}

Shape plays an important role in visualizing the feature and is one of the simple methods for image content description. Image retrieval with the help of shape is measuring the similarity between the shapes that are represented by their features. Shape based image retrieval requires two steps: feature extraction and similarity measurements amongst the extracted features.

\section{c. Texture}

Texture is another important property of the images. It is one of the important characteristics which is used to classify and recognize the objects and it is used for finding the similarities among the images in the dataset or databases. Texture of its own does not have the proficiency of finding similar images, but it can use the textured images from the non-textured ones and then it can combine with another visual attributes to make it more effective.

\section{3) SVM processing}

SVM is a supervised classification model. It needs training to do the classification task.SVM is one of the best-known methods in image retrieval process. A support vector machine (SVM) is a method of machine learning that attempts to take input data and classify it into one of the categories.SVM takes the training inputs, maps them into multidimensional space, then uses regression to find a hyper plane. It is essentially an input/output machine. A user is able to put in an input, and based on the model developed through training, it will return an output.SVM in our method will match features of dataset with query image and based on that image will be retrieved.

\section{4) Image Retrieval}

Dataset is eventually used by the SVM in the form of feature vectors. Feature vectors contain information about categories of image present in a dataset. So by using a sample of feature vectors as our training data, our classification system will be able to classify new examples of feature vectors which were not used in the design by SVM accurately. Also in case if the features are unprecedented to SVM, there will be an option to create new dataset.

\section{WORKFLOW}

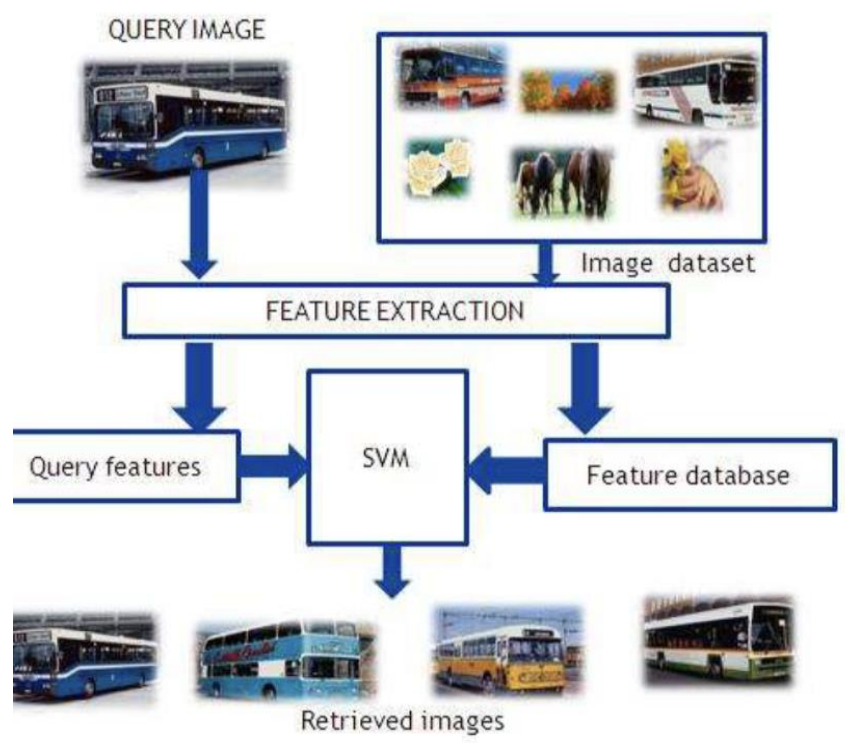

Figure(a): Image Retrieval Process

\section{DESCRIPTION:}

In this method 'Retrieval of images using SVM', features such as color, shape, edge, texture etc. will be extracted from the query image. These features are represented in the form of feature vectors. There will be a dataset. This dataset will contain features of selected images that is previously created by the user. To enable SVM to match features of query image and images of database, dataset has to be loaded into the software. These extracted features will be matched with the features of the dataset using SVM. SVM will then process features of the query image given by the user and dataset. Depending upon which the query image will be classified into corresponding category. The corresponding images from the database will be retrieved that feature wise resembles the same as that of the query image.

In above diagram a query image of a bus has given as an input to the feature extractor. Feature extractor extracts features depending upon the method used. These features and the dataset are then given to SVM for the features matching purpose. Based on the features that have matched with the features of the images present in the database, number of images will be retrieved as shown in the figure. 


\section{SNAPSHOTS}

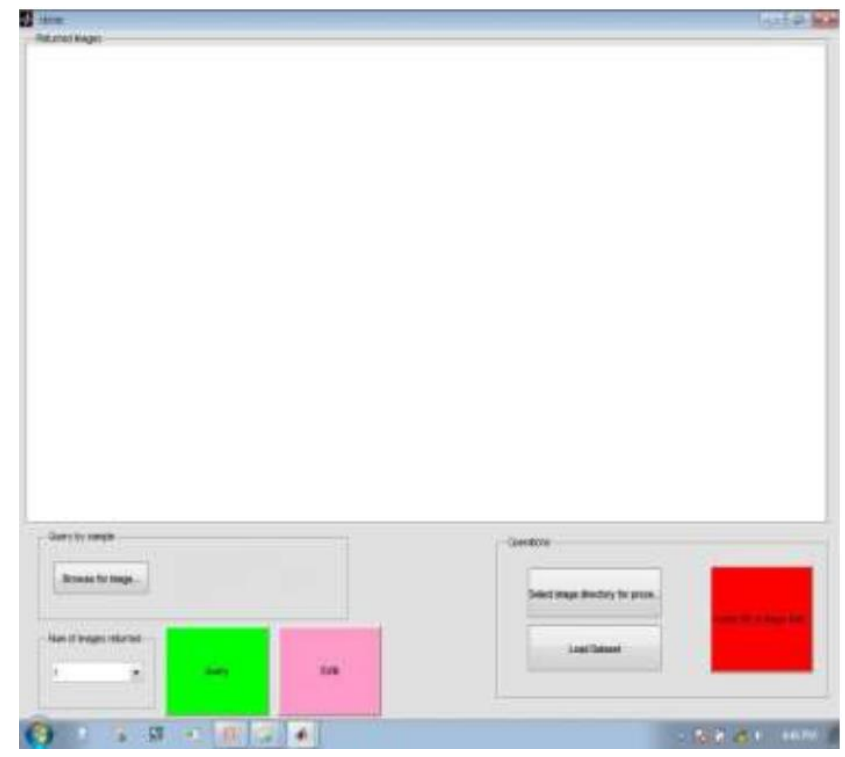

Figure (b): Snapshot of GUI

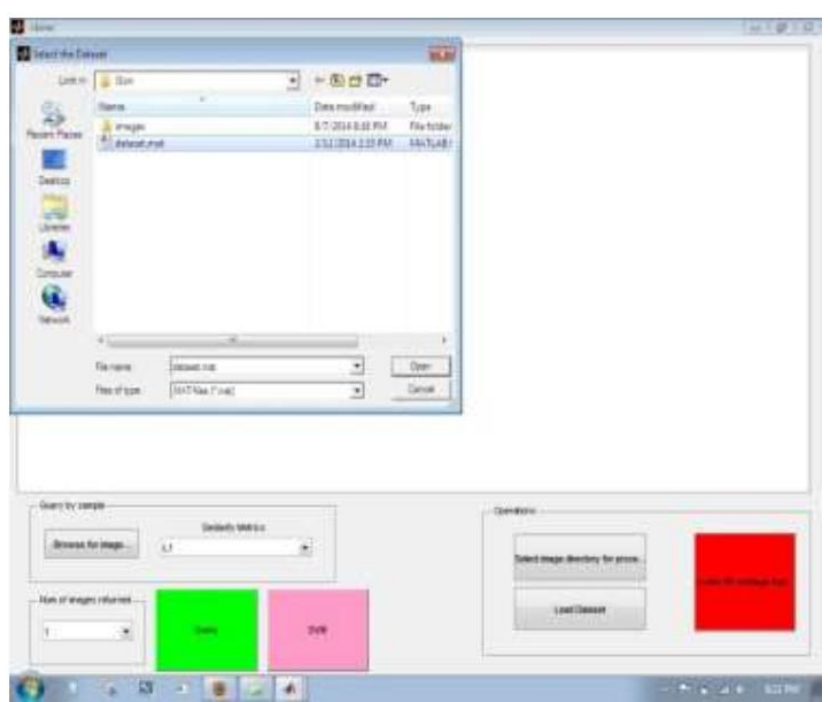

Figure(c): Snapshot before loading the dataset

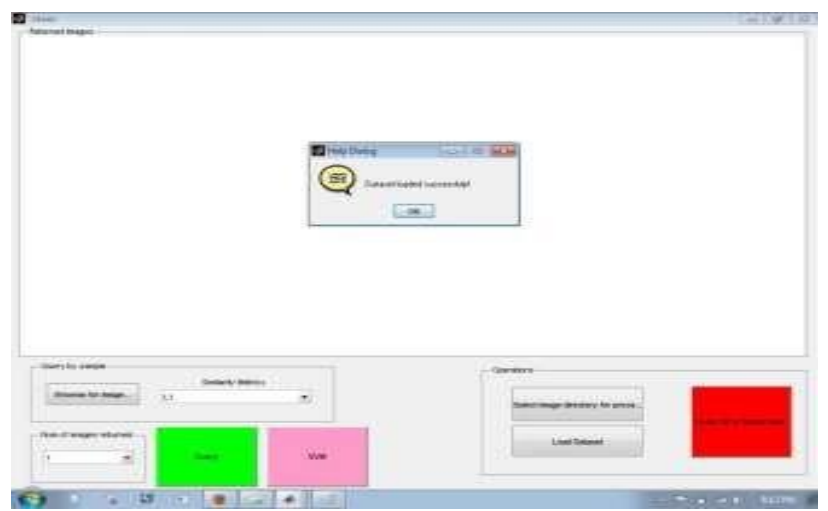

Figure (d): Snapshot after loading 

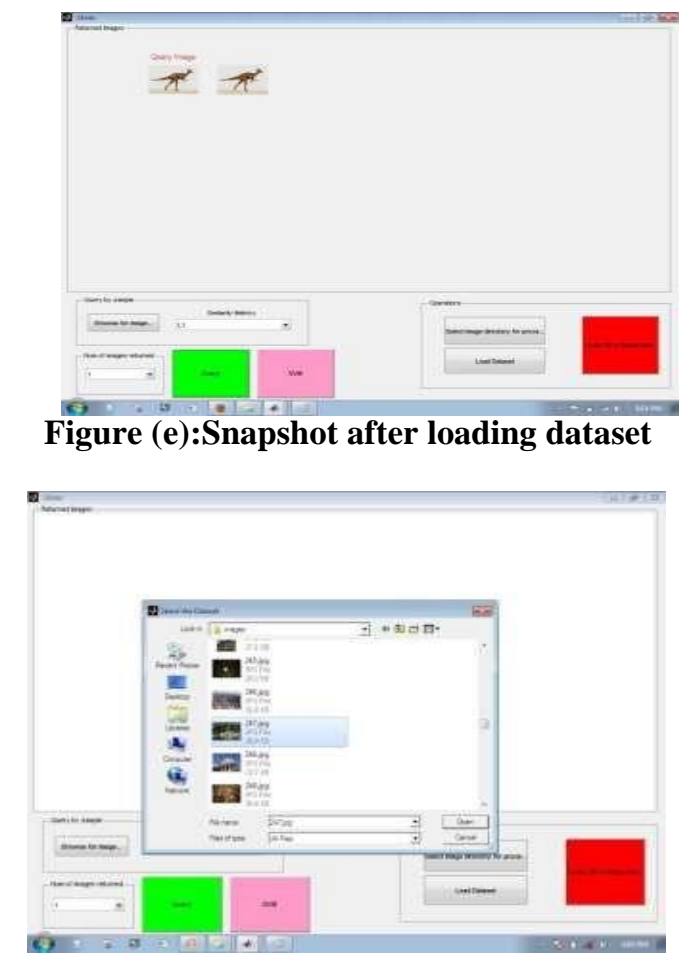

Figure (f): Snapshot after query is fired

\section{APPLICATIONS}

1. Remote Sensing:

Images captured by satellite can be searched and retrieved according to desired specification.

\section{Robotic Vision:}

Things that a robot will come across can be identified by taking pictures.

\section{Medical Applications:}

Various diagnoses can be performed by taking different images of the affected areas.

\section{Earth Sciences:}

Physical features of earth can be studied using this image retrieval technique.

\section{Art Collection:}

Arts like antique paintings can be particularly identified.

\section{Image searching in browser:}

Many images can be retrieved using search image option of browser

\section{CONCLUSION}

From this paper, one can conclude that the system is able to load the dataset successfully as well as query image can be given to the system. Apart from this, user can select how many numbers of images user wants to retrieve.

\section{FUTURE SCOPE}

The above review paper can be implementedinvarious aspects. Itcan create a larger dataset of more than 1000 images. The other future scope of this paperis the road extraction. This system can becollaboratedwith any website to make the tasks easier to comprehend and user friendly.

\section{REFERENCES}

[1]. Sumiti Bansal, Er. Rishamjot Kaur, "A Review on Content Based Image Retrieval using SVM", International Journal of Advanced Research in Computer Science and Software Engineering, Volume 4, Issue 7, July 2014

[2]. Neera Lal, Neetesh Gupta, Amit Sinhal , “A Review of Image Classification Techniques in Content Based Image Retrieval", Neera Lal et al, / (IJCSIT) International Journal of Computer Science and Information Technologies, Vol. 3 (5), 2012.

[3]. N. Kumaran, Dr. R. Bhavani, “ Texture and Shape Content Based MRI Image Retrieval System”, International Journal of Innovative Research in Science, Engineering and Technology, Volume 3, Special Issue 1, February 2014 
[4]. R.Ravinder Reddy, B.Kavya, Y Ramadevi, Ph.D., "A Survey on SVM Classifiers for Intrusion Detection”, International Journal of Computer Applications (0975 - 8887), Volume 98- No.19, July 2014 .

[5]. Amanbir Sandhu, Aarti Kochhar, "Content Based Image Retrieval using Texture, Color and Shape for Image Analysis", International Journal of Computers \& Technology Volume 3, No. 1, AUG, 2012

[6]. S. Mangijao Singh , K. Hemachandran, "Content Based Image Retrieval using Color Moment and Gabor based images using color movement and classification of brain computed tomography images", IET Image Processing 2012.

[7]. Reshma Chauudhari and A.M Patil "Content Based Image Retrieval Using Color and Shape Features", International Journal of Advanced Researchin Electrical, Electronics and Instrumentation Engineering, vol.1, 2012.

[8]. P.S. Malge et.al "Performance Evaluation of Texture based Image Retrieval",InternationalJournal ofComputer Applications (0975-8887) Volume 72-No.2, May 2013

[9]. RamadassSudhir et. Al, "A Efficient Content based Image Retrieval System using GMM and Relevance Feedback", International Journal of ComputerApplications (0975 -8887) Volume72-No.22, June 2013.

[10]. A. Padma Nanthagopal, R. Sukanesh,"Wavelet statistical texture features-based segmentation and classification of brain computed tomography images",IET Image Processing 2012.

[11]. Aditi Mehta,"Review and Comparison of Various Feature Extraction Techniques in CBIR", InternationalJournal of Computer Applications (0975-8887) Volume 71-No.23, June 2013.

[12]. D.Saraswathi,Chanemouga Priya, G.Sharmila, "An Efficient Color Feature Extraction Method for Content Based Image Retrieval”, NCWCAPS\|13, pg: no: 7-11,2013 